\title{
A Rapid Growth-Independent Antibiotic Resistance Detection Test by SYBR Green/Propidium lodide Viability Assay
}

\author{
Jie Feng ${ }^{1 \dagger}$, Rebecca Yee ${ }^{1 \dagger}$, Shuo Zhang ${ }^{1}$, Lili Tian', Wanliang Shi', Wen-Hong Zhang ${ }^{3}$ \\ and Ying Zhang ${ }^{1 *}$
}

'Department of Molecular Microbiology and Immunology, Bloomberg School of Public Health, Johns Hopkins University, Baltimore, MD, United States, ${ }^{2}$ Beijing Research Institute for Tuberculosis Control, Beijing, China, ${ }^{3}$ Key Laboratory of Medical Molecular Virology, Department of Infectious Diseases, Huashan Hospital, Shanghai Medical College, Fudan University, Shanghai, China

OPEN ACCESS

Edited by:

Marc Jean Struelens,

European Centre for

Disease Prevention and

Control, Sweden

Reviewed by:

Karsten Becker,

Universität Münster, Germany

Dimitris Tsaltas,

Cyprus University of

Technology, Cyprus

*Correspondence:

Ying Zhang

yzhang@jhsph.edu

tThese authors have contributed equally to this work.

Specialty section: This article was submitted to Infectious Diseases Surveillance, Prevention and Treatment,

a section of the journal

Frontiers in Medicine

Received: 12 November 2017 Accepted: 17 April 2018

Published: 03 May 2018

Citation:

Feng J, Yee $R$, Zhang S, Tian L, Shi W, Zhang $W-H$ and Zhang $Y$ (2018) A Rapid Growth-Independent Antibiotic Resistance Detection Test by SYBR Green/Propidium lodide Viability Assay.

Front. Med. 5:127. doi: 10.3389/fmed.2018.00127
Antibiotic-resistant bacteria have caused huge concerns and demand innovative approaches for their prompt detection. Current antimicrobial susceptibility tests (AST) rely on the growth of the organisms which takes 1-2 days for fast-growing organisms and several weeks for slow growing organisms. Here, we show for the first time the utility of the SYBR Green I/propidium iodide (PI) viability assay for rapidly identifying antibiotic resistance in less than 30 min for major, antibiotic-resistant, fast-growing bacteria, such as Staphylococcus aureus, Escherichia coli, Klebsiella pneumoniae, and Acinetobacter baumannii for bactericidal and bacteriostatic agents and in $16 \mathrm{~h}$ for extremely rapid detection of drug resistance for isoniazid and pyrazinamide in slow-growing Mycobacterium tuberculosis. The SYBR Green I/PI assay generated rapid and robust results in concordance with traditional AST methods. This novel growth-independent methodology changes the concept of the current growth-based AST and may revolutionize current drug susceptibility testing for all cells of prokaryotic and eukaryotic origin and, subject to further clinical validation, may play a major role in saving lives and improving patient outcomes.

Keywords: antibiotic resistance, antimicrobial susceptibility testing, SYBR Green/PI availability assay, Mycobacterium tuberculosis, Staphylococcus aureus, CRE bacteria

\section{INTRODUCTION}

A rising public health concern is the increasing emergence and spread of antibiotic-resistant bacteria (1-5). To combat antibiotic resistance, health-care providers are encouraged to minimize the unnecessary usages of antibiotics $(6,7)$. Hence, laboratory antibiotic or drug susceptibility testing results are imperative in providing health-care providers the knowledge to tailor the appropriate treatment to each individual $(8,9)$. However, currently, the common drug susceptibility tests (DST) all rely on growth of the organisms and take at least $18-24 \mathrm{~h}$ for non-fastidious organisms (10) and can be up to 1 month for fastidious slow growing organisms like Mycobacterium tuberculosis (11) before any information can be obtained about the pathogen's susceptibility or resistance to a particular drug, putting the patient's life at risk with potential worsened disease outcome and increased spread of antibiotic-resistant organisms. Here, we developed a novel, growth-independent antibiotic susceptibility testing methodology that can produce rapid and robust results in less than $24 \mathrm{~h}$ for both slow and fast-growing organisms.

The current antimicrobial susceptibility tests (AST) such as the Kirby-Bauer disk diffusion test or broth dilution method to determine a drug's minimum inhibitory concentration (MIC) provide 
accurate results, but the major drawback of these tests is the lengthy time (at least $16 \mathrm{~h}$ ) required until results are produced even for non-fastidious organisms (10). Recently, a MALDI Biotyper antibiotic susceptibility test rapid assay (MBT-ASTRA) was developed and showed high sensitivity against $M$. tuberculosis but the turnaround time for drug susceptibility results still takes at least 6 days after the cultures flag positive (12). The development of the automated VITEK ${ }^{\circledR} 2$ and microdroplet growth assay using MALDI-TOF produced high sensitivity for AST, but these tests are still growth dependent and take at least 12 and $4 \mathrm{~h}$, respectively $(13,14)$. In the case of multidrug-resistant tuberculosis and extensively drug-resistant TB (XDR-TB), a period of 2-6 weeks is required to obtain drug susceptibility testing results, which increases the risk of the development and spread of drug-resistant TB (15). The current DST for pyrazinamide (PZA), a unique sterilizing persister drug that shortens treatment from 9-12 to 6 months, include the MGIT 960 or BacT/ALERT systems at $\mathrm{pH}$ 6.0 (16); both tests are unreliable and subject to frequent false resistance problem and thus not routinely performed (17-20).

To expedite the process of antimicrobial susceptibility testing, we are introducing a novel approach using SYBR Green I/ propidium iodide (PI) staining viability assay, which we initially developed for quantifying the viability of and drug evaluation for Borrelia burgdorferi (21). The SYBR Green I dye is commonly used in molecular biology to stain nucleic acids and was used for viability assessment of bacteria by flow cytometry (22) but has not been used for AST. SYBR Green I is a permeable dye that stains all live cells green, whereas PI is an impermeant dye that stains only dead or damaged cells red with a compromised cell membrane (21). Hence, the green/red fluorescence ratio as easily measured by fluorescence microscopy or fluorescence microplate readers represents the viability of a bacterial population. As our assay is not growth-dependent, we have eliminated a bottleneck in the current AST.

Here, we demonstrate that the SYBR Green I/PI assay adapted from our previous study (21) can successfully be used for extremely rapid AST for antibiotic-resistant bacteria of different species in $30 \mathrm{~min}$ for fast-growing organisms including multidrug-resistant Staphylococcus aureus, Escherichia coli, Acinetobacter baumannii, and Klebsiella pneumoniae, and in $16 \mathrm{~h}$ for slow growing $M y$. tuberculosis, producing susceptibility results in concordance with traditional AST methods as provided by the CLSI guidelines. The SYBR Green I/PI assay overcomes not only the inefficiency of conventional culture or growth-based DST but also the limitations and compromised sensitivity of molecular testing methods that rely on detection of mutations that are associated with resistance (23).

\section{MATERIALS AND METHODS}

\section{Culture Media, Chemicals, and Antibiotics}

Staphylococcus aureus, E. coli, K. pneumoniae, A. baumannii cultures were cultivated in tryptic soy broth and tryptic soy agar (Becton Dickinson) and $M$. tuberculosis cultures were cultivated in $7 \mathrm{H} 9$ medium at $37^{\circ} \mathrm{C}$ with the appropriate antibiotics. S. aureus strains USA300, CA-409, NY315, CA127, E. coli strains W3110, UTI89, CFT073, and KTE181, K. pneumoniae (Isolate 7), A. baumannii isolate, and M. tuberculosis H37Ra, were obtained from ATCC (Manassas, VA, USA). Stock solutions of the antibiotics ampicillin, chloramphenicol, gentamicin, erythromycin, trimethoprim, ciprofloxacin, streptomycin, ceftriaxone, and cefotaxime (Sigma-Aldrich Co.) were prepared and sterilized through filtration and used at indicated concentrations. Antibiotic susceptibility assays for different bacteria were performed using Mueller-Hinton broth and agar per CLSI methods as described below.

\section{Conventional Antibiotic Susceptibility Tests}

The Kirby-Bauer disk diffusion assay was used to determine the susceptibility of the various strains to antibiotics. Instructions and also interpretation of zone diameters were made using the established standards as listed in the Clinical and Laboratory Standards Institute (10). Briefly, bacterial cultures were adjusted to a $0.5 \mathrm{McF}$ arland turbidity standard and spread onto MuellerHinton agar. Paper disks were impregnated onto the agar and the antibiotics at concentrations recommended by CLSI were added. The plates were incubated at $37^{\circ} \mathrm{C}$ overnight before the zone of inhibition was measured. The protocol used to test for antibiotic susceptibility using a broth dilution method was based on the recommendations of CLSI. Briefly, bacterial cultures were adjusted to a $0.5 \mathrm{McF}$ arland turbidity standard in Mueller-Hinton broth and drug concentrations of twofold dilutions.

\section{SYBR Green I/PI Assay}

SYBR Green I (10,000× stock, Invitrogen) was mixed with PI (20 mM, Sigma) in distilled $\mathrm{H}_{2} \mathrm{O}$. The staining dye for $S$. aureus, E. coli, A. baumannii, and $M$. tuberculosis were made by mixing SYBR Green I to PI (1:3) in $100 \mu$ distilled $\mathrm{H}_{2} \mathrm{O}$ (21). The staining dye for $K$. pneumoniae was made by mixing SYBR Green I to PI (3:1) in $100 \mu \mathrm{l}$ distilled $\mathrm{H}_{2} \mathrm{O}$. The SYBR Green I/ PI staining mix $(10 \mu \mathrm{l})$ was added to each $100 \mu \mathrm{l}$ of each sample. The sample was vortexed and incubated at room temperature in the dark for $20 \mathrm{~min}$. The green and red fluorescence intensity was detected using a Synergy H1 microplate reader by BioTek Instruments (VT, USA) at excitation wavelength of $485 \mathrm{~nm}$ and $538 \mathrm{~nm}$ and $612 \mathrm{~nm}$ for green and red emission, respectively. The percentage of live cells in each sample was determined by a regression equation generated by a standard curve. To generate a standard curve, different proportions of live and isopropyl alcohol killed cells were made. The staining mixture was added to each sample and the green/red fluorescent ratios were measured as described above. The regression equation was generated using the least-square fitting analysis. Specimens of $S$. aureus and K. pneumoniae were examined on the Keyence BZ-X710 Fluorescence Microscope and images were recorded and processed using BZ-X Analyzer provided by Keyence (Osaka, Japan). Specimens of $M$. tuberculosis cells were examined on a Zeiss AxioImager M2 microscope equipped with epifluorescence illumination. Pictures were taken using ORCA- ${ }^{2}$ high resolution digital camera (HAMAMATSU, Japan). Image 
Pro-Plus software was used to quantitatively determine the fluorescence intensity.

\section{Isolation of Spontaneous Isoniazid- Resistant and PZA-Resistant Mutants of $M$. tuberculosis}

Mycobacterium tuberculosis H37Ra was grown in 7H9 liquid medium (Difco) supplemented with $0.05 \%$ Tween 80 and $10 \%$ bovine serum albumin-dextrose-catalase (ADC) enrichment at $37^{\circ} \mathrm{C}$ for approximately $10-14$ days (mid- to late-log phase). PZA (Sigma-Aldrich Co.) or isoniazid (INH) was dissolved in deionized water at a stock concentration of $10 \mathrm{mg} / \mathrm{ml}$ and filtersterilized and incorporated into $7 \mathrm{H} 11$ agar plates containing ADC at concentrations of $100-300 \mu \mathrm{g} / \mathrm{ml}, \mathrm{pH} 6.0$ for PZA, or at $0.2-2 \mu \mathrm{g} / \mathrm{ml}$ for INH. Mutants that grew on the PZA or INH containing plates after 3 - to 4 -week incubation at $37^{\circ} \mathrm{C}$ were picked and grown in $7 \mathrm{H} 9$ liquid medium to confirm for resistance. The PZA susceptibility testing of the PZA-resistant mutants was performed on $7 \mathrm{H} 11$ agar plates containing $100-300 \mu \mathrm{g} / \mathrm{ml}$ PZA (pH 6.0) as described (24).

\section{SYBR Green I/PI Viability Staining for Rapid Antibiotic Susceptibility Testing}

Cultures of S. aureus, E. coli, and K. pneumoniae were grown to stationary phase and diluted OD600 of 0.1 (approx. $5 \times 10^{7}$ ) before treatment with antibiotics of indicated concentrations. After $30 \mathrm{~min}$ of incubation at $37^{\circ} \mathrm{C}$, the cultures were stained with SYBR Green I/PI and the percentage of viable cells was determined as described above. For rapid drug susceptibility testing of M. tuberculosis, the culture of parental strain H37Ra and INH-resistant mutant strains were, respectively, added into a 96-well plate, each well containing $80 \mu$ of culture. INH in $20 \mu \mathrm{l}$ $7 \mathrm{H} 9$ medium was added into $80 \mu \mathrm{l}$ culture as the INH-treated group; meanwhile, the untreated group was the control. The plate was sealed by aluminum seal foil and incubated at $37^{\circ} \mathrm{C}$ overnight $(15 \mathrm{~h})$, followed by the addition of $10 \mu \mathrm{l} \mathrm{SYBR}$ green I/ PI staining mixture to the above treated and untreated cultures. The plate was incubated at room temperature in the dark for $45 \mathrm{~min}$ followed by fluorescence detection with a microplate reader as described above. Relative to the untreated group, the viability of INH-treated H37Ra and INH-resistant mutants were calculated according to the green and red fluorescence ratio. The difference of residual viability between H37Ra WT and INHresistant mutants were compared. For PZA susceptibility testing in M. tuberculosis, $100 \mu \mathrm{l}$ of 20-day-old cultures of M. tuberculosis H37Ra and PZA-resistant mutants (approx. $5 \times 10^{6}$ ) was mixed with $4 \mu \mathrm{l} \mathrm{PZA}(50 \mathrm{mg} / \mathrm{ml})$ alone or with enhancer [salicylic acid (SA) $(40 \mu \mathrm{g} / \mathrm{ml})]$. After overnight incubation, SYBR Green I/PI staining dye was added, incubated at room temperature in the dark for $45 \mathrm{~min}$, and the percentage of viable cells was determined as described above. All untreated groups were used as controls.

\section{Statistical Analyses}

Statistical analyses were performed using two-tailed Student's $t$-test and two-way ANOVA, where appropriate. Mean differences were considered statistically significant if $p$ was $<0.05$.
The proportion of dead cells with their respective 95\% confidence intervals was determined using the results from repeated testing from the SYBR Green I/PI assay. All experiments were performed in triplicates. Analyses were performed using GraphPad Prism and Microsoft Office Excel.

\section{RESULTS}

\section{SYBR Green I/PI Assay Can Assess the Viability of Various Bacterial Pathogens}

To develop a rapid test to evaluate antimicrobial susceptibility in the clinic, it is crucial to ensure that SYBR Green I/PI test can assay the viability for a range of test organisms, especially those considered by the CDC as serious or urgent threats. Hence, we applied the SYBR Green I/PI assay to generate a standard curve for representative Gram-positive bacteria $S$. aureus, Gram-negative bacteria K. pneumoniae, A. baumannii, and E. coli, and acid-fast $M$. tuberculosis. We adapted the SYBR Green I/PI assay which we developed for assessing the viability of B. burgdorferi (21) for the above organisms. We grew the bacteria in their normal culture media and to generate dead cells, we killed the bacteria with incubation of 70\% isopropyl alcohol for $1 \mathrm{~h}$. After staining the cells with SYBR Green I/PI, we measured the green and red fluorescence intensities using a fluorescence microplate reader (BioTek Synergy HT) and generated a standard curve showing the relationship of bacterial viability and the percentages of green (live) and red (dead) cells. A linear relationship between the green/red fluorescence ratios and the percentages of live $S$. aureus, K. pneumoniae, E. coli, A. baumannii, and M. tuberculosis with $R^{2}$ values of $0.9934,0.9980,0.9853,0.9807$, and 0.9561 , respectively, were generated (Figures 1A-E). Additionally, staining of varying viable proportions $(0,50$, and $100 \%)$ of Gram-positive $S$. aureus (Figure 1F) and Gram-negative K. pneumoniae samples (Figure 1F) with SYBR Green I/PI revealed a good correlation between the number of viable cells determined by fluorescence microscopy imaging and the actual known viable percentages determined by the fluorescence microplate reader.

\section{Development of a Rapid DST Using SYBR Green I/PI Assay for Fast-Growing Organisms}

Because resistant organisms are more difficult to kill than susceptible organisms, the SYBR Green I/PI assay can distinguish resistant and susceptible categories based on the amount of residual viable cells after drug treatment (25). Since the SYBR Green I/PI assay detects viability and killing, we hypothesize that this assay will eliminate the time needed for bacterial growth and produce AST results in a shorter time than the current growth-based methods. To test this hypothesis and ensure that our assay produced results that are in concordance with the standards provided by CLSI, we evaluated clinical isolates of different bacterial species for their susceptibility categories against drugs based on the conventional Kirby-Bauer disk diffusion assay (10). Next, to identify an appropriate time where viability differences between resistant and sensitive strains were observed, we measured the green/red fluorescence ratios of both sensitive 

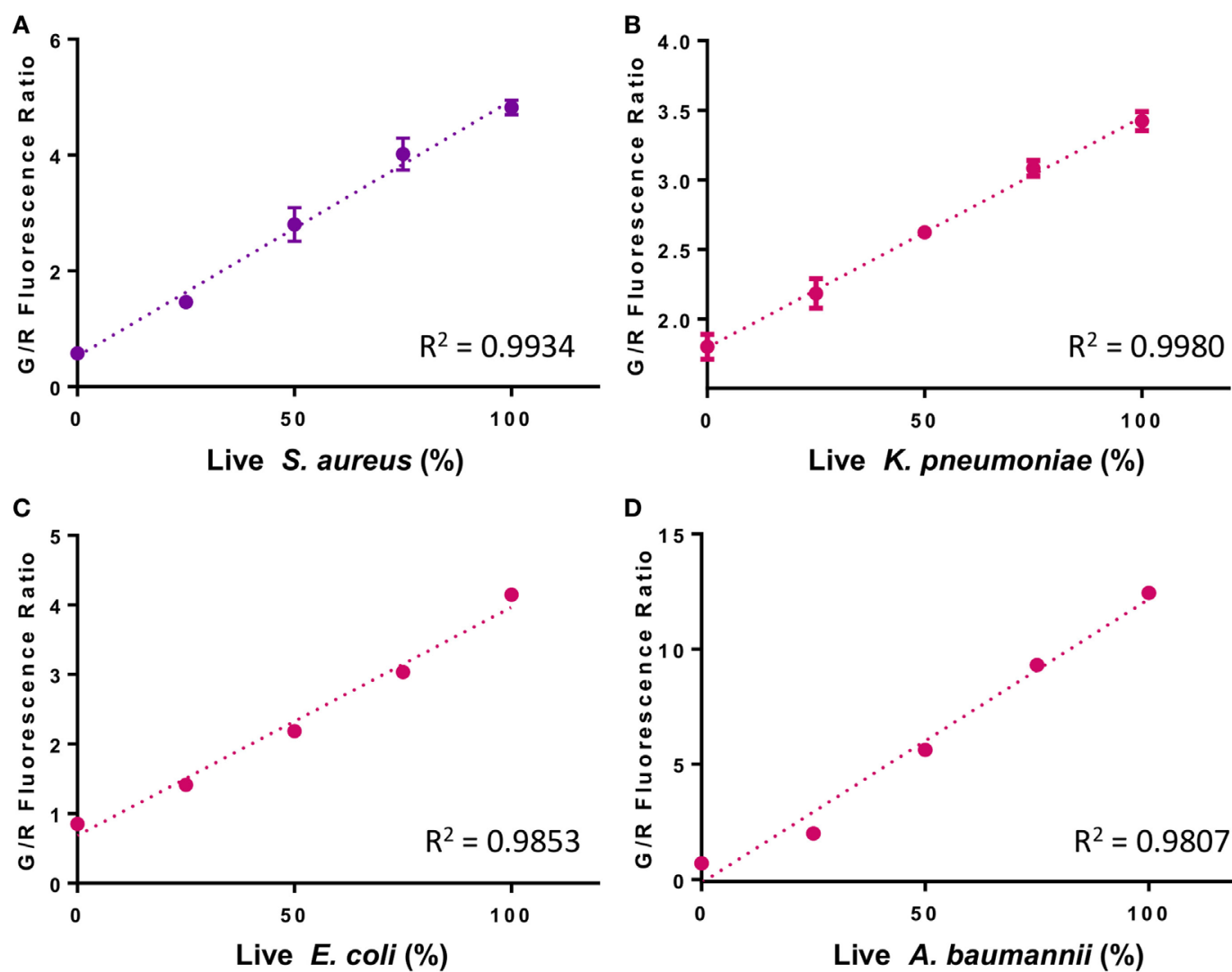

Live K. pneumoniae (\%)

D

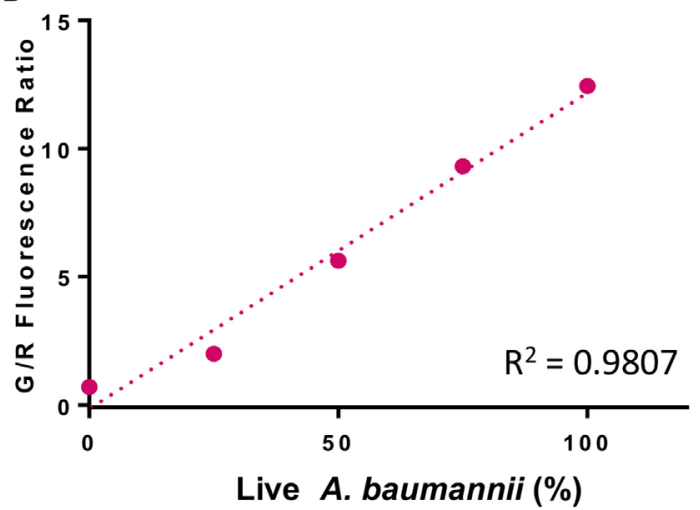

E

$\mathbf{F}$
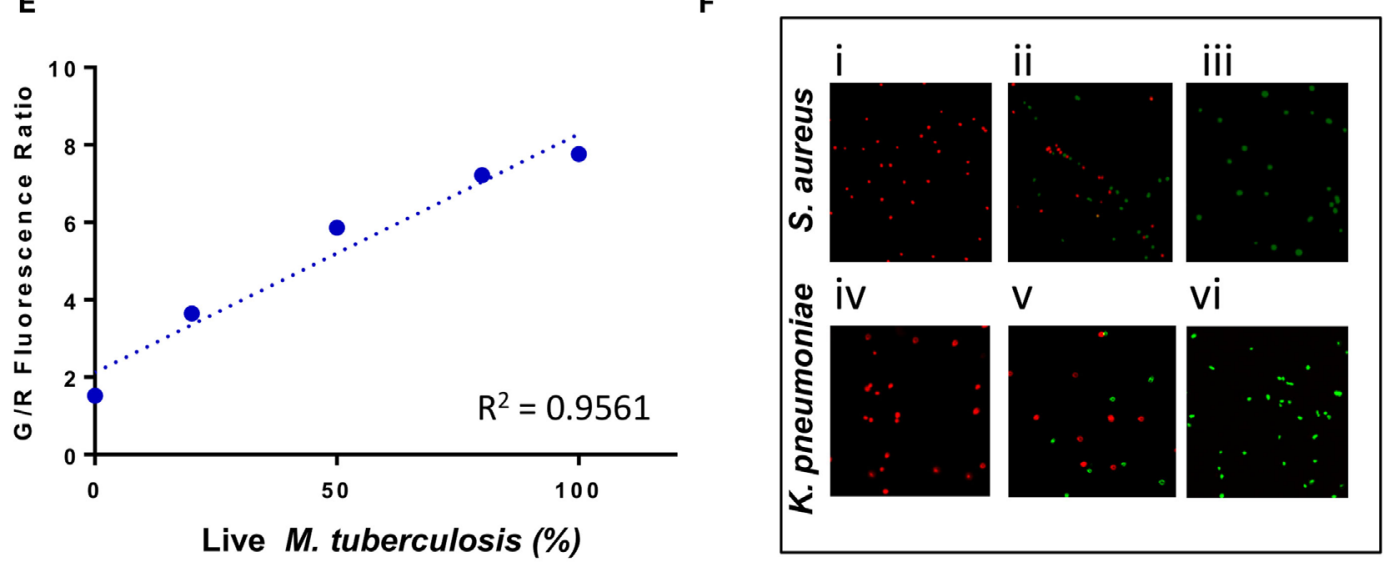

FIGURE 1 | A linear relationship between the percentage of live cells and the green/red fluorescence ratio from the SYBR Green I/propidium iodide (PI) viability assay for different bacterial species. Known proportions of isopropyl killed (30 min) and live (A) Staphylococcus aureus (USA300), (B) Klebsiella pneumoniae (Isolate 7),

(C) Escherichia coli (W3110), (D) Acinetobacter baumannii, and (E) Mycobacterium tuberculosis (H37Ra) were stained with SYBR Green I/PI and measured using a fluorescence plate reader. A linear regression line was determined. (F) Fluorescence microscopy image showing known proportions (0, 50, and 100\%) of live (i-iii) S. aureus (USA300) and (iv-vi) K. pneumoniae (isolate 7) stained with SYBR Green I/PI reveal red (dead) and green (live) ratios in concordance with the known proportions of live and killed organisms from representative Gram-positive (S. aureus) and Gram-negative (K. pneumoniae) bacteria. Data represent the means \pm SEMs.

and resistant strains across $1.5 \mathrm{~h}$ of drug exposure time. For $S$. aureus, a significant decrease in viability during kanamycin exposure $(100 \mu \mathrm{g} / \mathrm{ml})$ was observed as early as $30 \mathrm{~min}$ between the sensitive Newman strain and the resistant CA127 clinical strain (Figure 2A). For E. coli, we also observed a significant decrease in viability in sensitive W3110 strain and resistant KTE181 strain after exposure to ampicillin $(100 \mu \mathrm{g} / \mathrm{ml})$ for $30 \mathrm{~min}$ (both, twoway ANOVA, $p<0.005$ ) (Figure 2B).

To optimize the conditions for our SYBR Green/PI assay, we exposed $S$. aureus strains to a panel of bactericidal and bacteriostatic drugs of various concentrations. To establish a quantitative cutoff distinguishing resistant and susceptible 




FIGURE 2 | SYBR Green I/propidium iodide monitors the dynamics of resistant and sensitive strains during antibiotic drug exposure. A significant decrease in green/red fluorescence ratio was seen as early as $30 \mathrm{~min}$ between (A) sensitive Staphylococcus aureus strain Newman and resistant strain CA127 during kanamycin exposure and (B) sensitive Escherichia coli strain W3110 and resistant strain KTE181 during ampicillin exposure (twoway ANOVA, ${ }^{\star \star \star} p<0.0005$ ). Data represent the means \pm SEMs.

strains, we utilized a mathematical formula to determine the proportion of cells killed based on the green and red fluorescence values from microtiter plate readings. The proportion of cells killed was calculated by the formula $\left(\mathrm{LD}_{\text {treated }}-\mathrm{LD}_{\text {untreated }}\right) /$ $\mathrm{LD}_{\text {untreated, }}$ where LD is equal to the ratio of live (green fluorescence) and dead (red fluorescence) cells. We chose a cutoff of approximately $20 \%$ of killed cells (between resistant and sensitive strains) which produced statistically significant results (Student's $t$-test, $p<0.05$ ) that were in concordance with susceptibility results based on the interpretive criteria from the conventional Kirby-Bauer method and broth dilution method as published in the CLSI guidelines (Table 1). After $30 \mathrm{~min}$, gentamicin $(200 \mu \mathrm{g} / \mathrm{ml})$ killed $1 \%$ of gentamicin-resistant clinical isolate CA127 but $12-26 \%$ in sensitive Newman and USA300 strains (Figure 3A). Kanamycin $(100 \mu \mathrm{g} / \mathrm{ml})$ killed $7 \%$ of CA127 and $0.5 \%$ of NY315 but over $30 \%$ of Newman (Figure 3B). Erythromycin $(200 \mu \mathrm{g} / \mathrm{ml})$ killed $2 \%$ of NY315 but $26 \%$ of sensitive control Newman strain (Figure 3C). Ciprofloxacin $(100 \mu \mathrm{g} / \mathrm{ml})$ killed $15 \%$ of CA127 and $45 \%$ of Newman strain (Figure 3D). All differences were statistically significant (Student's $t$-test, $p<0.05$ ).

Then, we performed AST for various Gram-negative pathogens including E. coli and K. pneumoniae. For E. coli, upon ampicillin treatment $(100 \mu \mathrm{g} / \mathrm{ml})$, sensitive strains W3110 and UTI89 were killed 53 and 40\%, respectively, while resistant strain KTE181 was not killed at all (Figure 4A). Trimethoprim treatment $(50 \mu \mathrm{g} / \mathrm{ml})$ killed $41 \%$ of sensitive strain W3110 but 28 and $6 \%$ of
TABLE 1 | Summary of broth dilution results and SYBR Green I/propidium iodide (PI) assay.

\begin{tabular}{|c|c|c|c|}
\hline $\begin{array}{l}\text { Broth dilution } \\
\text { CLSI minimum } \\
\text { inhibitory } \\
\text { concentration } \\
(\mu \mathrm{g} / \mathrm{ml})\end{array}$ & $\begin{array}{c}\text { SYBR } \\
\text { Green I/PI } \\
\text { Prop.dead } \\
\text { avg }(95 \% \mathrm{Cl})\end{array}$ & $\begin{array}{c}\text { CLSI } \\
\text { Interpretation }\end{array}$ & $\begin{array}{c}\text { SYBR } \\
\text { Green I/PI } \\
\text { Interpretation }\end{array}$ \\
\hline
\end{tabular}

$\begin{array}{lcccc}\begin{array}{l}\text { Escherichia coli } \\ \text { Ampicillin } \\ \text { W3110 }\end{array} & 2 & \begin{array}{c}53.9 \% \\ (40.6-67.1)\end{array} & \mathrm{S} & \mathrm{S} \\ \text { UT189 } & 2 & \begin{array}{c}34.4 \% \\ (0.08-68.7)\end{array} & \mathrm{S} & \mathrm{S} \\ \text { KTE181 } & 1,024 & 0 \%(0-0) & \mathrm{R} & \mathrm{R} \\ \text { Trimethoprim } & & 40.9 \% & \mathrm{~S} & \mathrm{~S} \\ \text { W3110 } & 1 & (34.8-47.0) & & \mathrm{S} \\ \text { CFT073 } & 1 & 27.9 \% & \mathrm{~S} & \mathrm{R} \\ \text { KTE181 } & & (24.9-31.0) & & \mathrm{R} \\ & 128 & 5.9 \% & & \end{array}$

\begin{tabular}{lcccc}
\hline $\begin{array}{l}\text { Staphylococcus aureus } \\
\text { Gentamicin } \\
\text { Newman }\end{array}$ & 4 & $\begin{array}{c}18.5 \% \\
(8.81-28.2) \\
1.3 \%\end{array}$ & $\mathrm{~S}$ & $\mathrm{~S}$ \\
CA127 & 16 & $\mathrm{R}$ & $\mathrm{R}$ \\
Kanamycin & & $(0-3.87)$ & & \\
Newman & 8 & $31.2 \%$ & $\mathrm{~S}$ & $\mathrm{~S}$ \\
CA127 & 16 & $\begin{array}{c}(16.7-45.6) \\
6.9 \%\end{array}$ & $\mathrm{~S}$ & $\mathrm{~S}$ \\
NV315 & 128 & $\begin{array}{c}(2.32-11.5) \\
0.18 \%\end{array}$ & $\mathrm{R}$ & $\mathrm{R}$ \\
Ciprofloxacin & & $(0-0.052)$ & & $\mathrm{S}$ \\
Newman & 0.13 & $45.3 \%$ & $\mathrm{~S}$ & $\mathrm{R}$ \\
CA127 & 8 & $(39.4-51.3)$ & & $\mathrm{R}$ \\
\hline
\end{tabular}

resistant strains CFT073 and KTE181, respectively (Figure 4B). Streptomycin treatment $(50 \mu \mathrm{g} / \mathrm{ml})$ killed $41 \%$ of sensitive strain W3110 but $1 \%$ for resistant strain KTE181 (Figure 4C). For K. pneumoniae upon ceftriaxone $(25 \mu \mathrm{g} / \mathrm{ml})$ (Figure 4D) and cefotaxime $(50 \mu \mathrm{g} / \mathrm{ml})$ (Figure $4 \mathrm{E})$ treatment, sensitive strain W3110 was killed 38 and 26\%, respectively, but the resistant isolate 7 strain was killed 5 and 4\%, respectively. All differences were statistically significant (Student's $t$-test, $p<0.05$ ). We did not observe any reaction between antibiotics tested and the SYBR Green I and PI dyes used, and dyes used at the testing concentrations did not significantly affect the viability of the bacteria in control experiments.

\section{Demonstration that Bacteriostatic Drugs Work as Well as Bactericidal Drugs With SYBR Green I/PI Assay in Distinguishing Sensitive and Resistant Strains}

By definition, bactericidal drugs kill the bacteria whereas bacteriostatic drugs inhibit the growth of the bacteria. Here, we wanted to address if the SYBR Green I/PI assay can detect sensitive and resistant strains of not only bactericidal drugs but bacteriostatic 

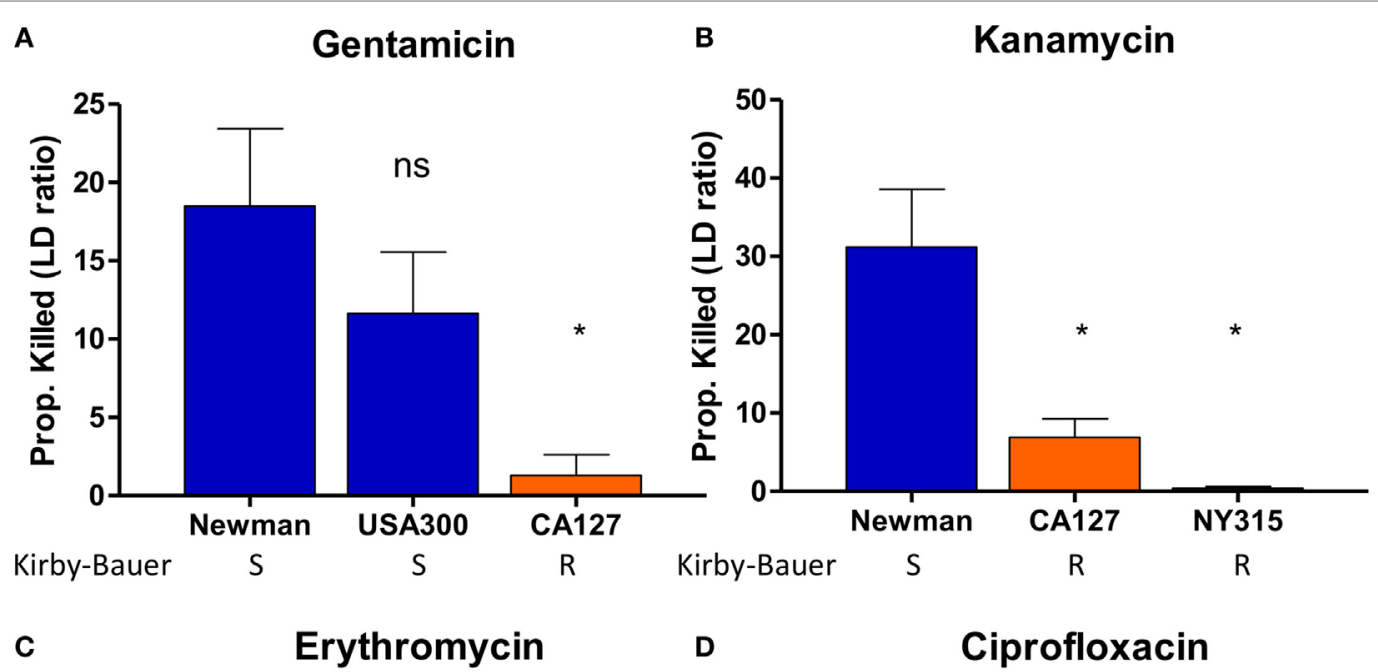

D Ciprofloxacin

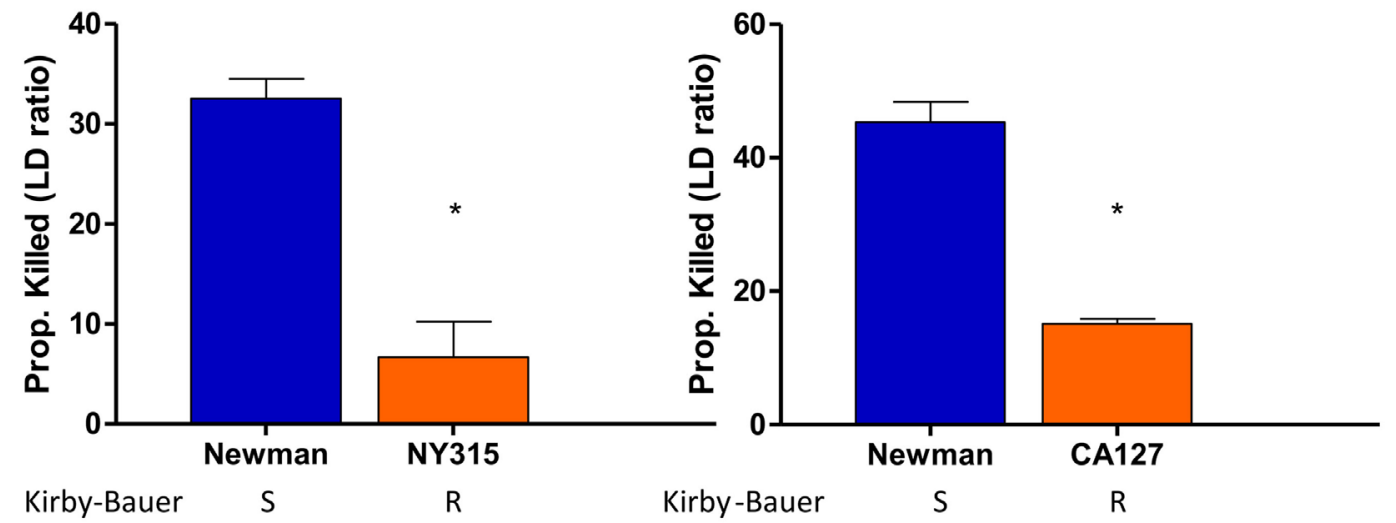

FIGURE 3 | SYBR Green I/propidium iodide (PI) stain can distinguish between R (resistant) and S (sensitive) strains of Staphylococcus aureus against various antibiotics in $30 \mathrm{~min}$. Treatment with (A) gentamicin $(100 \mu \mathrm{g} / \mathrm{ml})$, (B) kanamycin (100 $\mu \mathrm{g} / \mathrm{ml})$, (C) erythromycin (400 $\mu \mathrm{g} / \mathrm{ml})$, and (D) ciprofloxacin (100 $\mu \mathrm{g} / \mathrm{ml})$ was added to various overnight $S$. aureus strains diluted to 1:25 (OD600 $=0.1)$. After incubation with antibiotics for 30 min, SYBR Green $1 / P I$ staining was performed and distinguished the strains and their respective susceptibility categories. All susceptibility results were in concordance with results from the Kirby-Bauer disk diffusion test (Student's $t$-test, $\left.{ }^{\star} p<0.05\right)$. Data represent the means + SEMs.

drugs as well. Our SYBR Green I/PI assay revealed that treatment with increasing concentrations of bactericidal drug kanamycin for 30 min consistently killed $40 \%$ of the cells in the sensitive S. aureus Newman strain but an average of $2 \%$ in the resistant NY315 strain, as expected (Figure 5A). For E. coli, the percentage of killed cells after treatment with bactericidal drug ampicillin increased in a dose-dependent manner. A statistical significant difference between the sensitive strain W3110 and resistant strain KTE181 was evident using $100 \mu \mathrm{g} / \mathrm{ml}$ of ampicillin (Figure 5B).

To test if the SYBR Green I/PI assay can detect sensitive and resistant strains by bacteriostatic drugs, we added increasing concentrations of bacteriostatic agent erythromycin $(200-1,000 \mu \mathrm{g} / \mathrm{ml})$ for $S$. aureus strains (Figure 5C) and bacteriostatic drug trimethoprim $(50-300 \mu \mathrm{g} / \mathrm{ml})$ for E. coli strains (Figure 5D). In both cases, the proportion of dead cells (measured by green/ red fluorescence ratio) increased in a dose-dependent manner as determined by the SYBR Green I/PI staining. For both sensitive and resistant strains, all cells were killed as the concentrations of drugs increased. Significant difference between the amount of killed cells in sensitive $S$. aureus Newman and resistant NY315 strain (using $200 \mu \mathrm{g} / \mathrm{ml}$ erythromycin) and sensitive E. coli W3110 and resistant KTE181 strain (using $50 \mu \mathrm{g} / \mathrm{ml}$ trimethoprim) were observed at appropriate drug concentrations, though killing activity between the strains became insignificant when the highest concentration of drugs [erythromycin $(>400 \mu \mathrm{g} / \mathrm{ml})$ and trimethoprim $(>150 \mu \mathrm{g} / \mathrm{ml})$ ] was administered.

\section{Implementation of SYBR Green I/PI Assay for Rapid Drug Susceptibility Testing for M. tuberculosis}

To determine if this assay also works for slow growing M. tuberculosis, we tested the utility of the SYBR Green/PI assay for detecting isoniazid (INH) resistance. Because INH mainly acts on growing cells of $M$. tuberculosis (23) we used a 7- to 10-day log phase culture of M. tuberculosis H37Ra and INHresistant mutants (I2 and I4). We treated parental strain H37Ra and resistant mutants with different INH concentrations for 
A

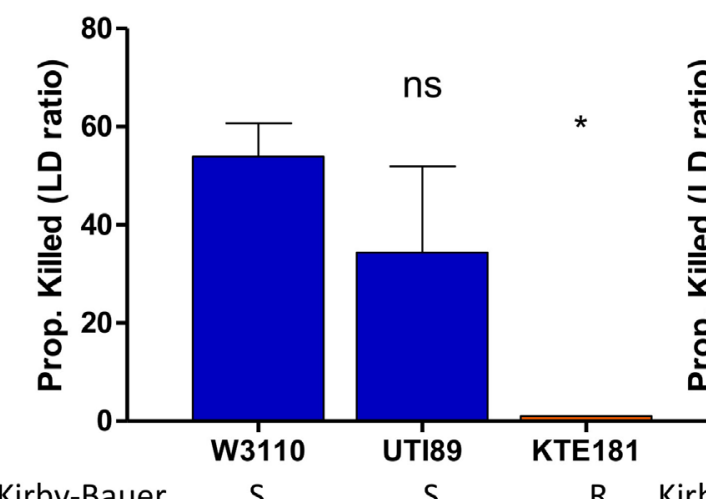

B
Trimethoprim

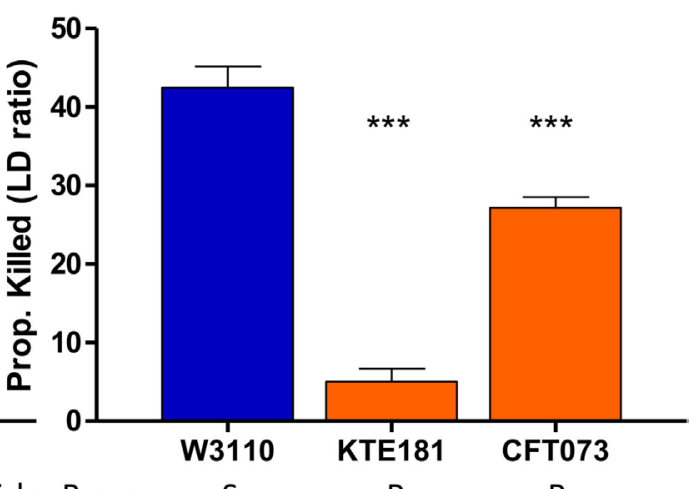

$\mathrm{R}$
C

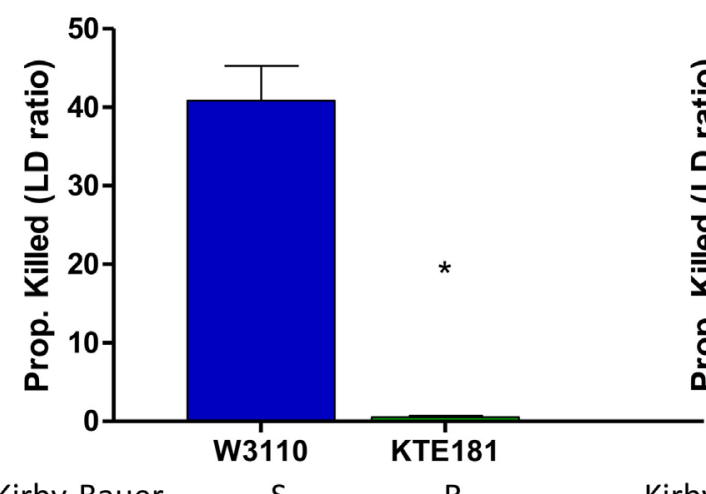

D

Ceftriaxone



E

Cefotaxime



FIGURE 4 | SYBR Green I/propidium iodide (PI) stain can distinguish between R (resistant) and S (sensitive) strains of Gram-negative pathogens against various antibiotics in $30 \mathrm{~min}$. Treatment with (A) ampicillin $(100 \mu \mathrm{g} / \mathrm{ml})$, (B) trimethoprim $(50 \mu \mathrm{g} / \mathrm{ml})$, and (C) streptomycin $(50 \mu \mathrm{g} / \mathrm{ml})$ was added to overnight cultures of Escherichia coli strains diluted to $1: 25(\mathrm{OD} 600=0.1)$. Treatment with $(\mathbf{D})$ ceftriaxone $(25 \mu \mathrm{g} / \mathrm{ml})$ and $(\mathbf{E})$ cefotaxime $(50 \mu \mathrm{g} / \mathrm{ml})$ was added to overnight cultures of Klebsiella pneumoniae strains diluted to 1:25 $(\mathrm{OD} 600=0.1)$. After incubation with antibiotics for $30 \mathrm{~min}$, SYBR Green I/PI staining was performed and distinguished their respective susceptibility categories. All susceptibility results were in concordance with results from the Kirby-Bauer disk diffusion test (Student's $t$-test, $\left.{ }^{*} p<0.05,{ }^{* *} p<0.05,{ }^{* * *} p<0.0005\right)$. Data represent the means + SEMs.

$16 \mathrm{~h}$, followed by the SYBR Green I/PI assay to determine the percentage of cells killed. No significant differences between the proportion of killed $\mathrm{H} 37 \mathrm{Ra}$ and INH-resistant strains at INH concentration of $10 \mu \mathrm{g} / \mathrm{ml}$ were observed. However, at higher concentrations of INH (500 and $1,000 \mu \mathrm{g} / \mathrm{ml}$ ), parental strain $\mathrm{H} 37 \mathrm{Ra}$ was killed more effectively compared to its resistant 



FIGURE 5 | SYBR Green I/propidium iodide (PI) assay reveals killing of bactericidal and bacteriostatic antibiotics in 30 min. SYBR Green I/PI assay reveals killing between sensitive and resistant strains after treatment with increasing concentrations of bactericidal drugs (A) kanamycin for Staphylococcus aureus and (B) ampicillin for Escherichia coli. Upon treatment with increasing concentrations of bacteriostatic drugs (C) erythromycin for S. aureus and (D) trimethoprim for E. coli, SYBR Green I/PI assay was consistently able to distinguish sensitive and resistant strains when administered at different concentrations for both sensitive and resistant strains (Student's $t$-test, ${ }^{\star} p<0.05,{ }^{* *} p<0.005,{ }^{* \star *} p<0.0005$ ). Data represent the means + SEMs.

mutants I2 and I4 (Figure 6A) $(p<0.005, p<0.05$, respectively). A high concentration of INH $(1,000 \mu \mathrm{g} / \mathrm{ml})$, also caused death of the INH-resistant strains reducing the difference between the sensitive parent strain and the resistant mutants (Figure 6A). These results indicate that the SYBR Green I/PI assay at the appropriate drug concentration $(500 \mu \mathrm{g} / \mathrm{ml})$ can rapidly detect INH resistance in $M$. tuberculosis in $16 \mathrm{~h}$.

We then went on to assess if the SYBR Green/PI assay could be used for detecting PZA resistance, the most challenging DST of all TB drugs (17-19). Because PZA plays a critical role in shortening TB treatment from 9-12 to 6 months and is used for treating both drug susceptible and drug-resistant $\mathrm{TB}$, accurate testing of PZA susceptibility is crucial for treatment outcome $(15,26)$. Unfortunately, the current PZA susceptibility testing methods often produce unreliable false positive results and can take weeks due to reliance on growth of $M$. tuberculosis $(17,19,20)$. Also, a small percentage of PZA-resistant strains do not have mutations in $p n c A$ or $r p s A$ involved in PZA's mechanisms of resistance, which limits the capacity of molecular methods for detecting PZA resistance (27). To evaluate if our rapid AST can detect PZA resistance, we treated parental strain M. tuberculosis H37Ra (MIC < $100 \mu \mathrm{g} / \mathrm{ml} \mathrm{PZA,} \mathrm{pH} \mathrm{5.9)}$ and PZA-resistant mutants $(>200 \mu \mathrm{g} / \mathrm{ml} \mathrm{PZA}, \mathrm{pH}$ 5.9) with different PZA concentrations followed by SYBR Green I/PI staining. We found that the difference in proportion of dead cells between the susceptible H37Ra and PZA-resistant strains increased with higher PZA concentration after overnight $(16 \mathrm{~h})$ treatment. After optimization, we found that susceptible H37Ra strain was killed more effectively compared to PZAresistant mutants with $2 \mathrm{mg} / \mathrm{ml}$ PZA treatment overnight (Figure 6B), suggesting that our assay could identify PZA resistance in just $16 \mathrm{~h}$.

Since PZA acts differently from common antibiotics by killing non-replicating $M$. tuberculosis persisters (27), certain weak acids such as SA and acid $\mathrm{pH}$ are known to enhance the activity of PZA against $M$. tuberculosis $(28,29)$. Therefore, we tested if weak acid SA added to the PZA treatment could show more significant difference in residual viability between the parental susceptible strain H37Ra and PZA-resistant mutants. Indeed, we found that SA $(40 \mu \mathrm{g} / \mathrm{ml})$ significantly enhanced the activity of PZA against parental strain $\mathrm{H} 37 \mathrm{Ra}$ but not PZA-resistant mutants at $2 \mathrm{mg} / \mathrm{ml} \mathrm{PZA} \mathrm{in} \mathrm{overnight}$ $(16 \mathrm{~h})$ treatment (Figure 6B). The proportion of dead H37Ra was significantly higher than the two PZA-resistant mutants P2 and P5 (Figure 6B) $(p<0.05)$. These results showed that the SYBR Green I/PI assay with weak acids could differentiate 


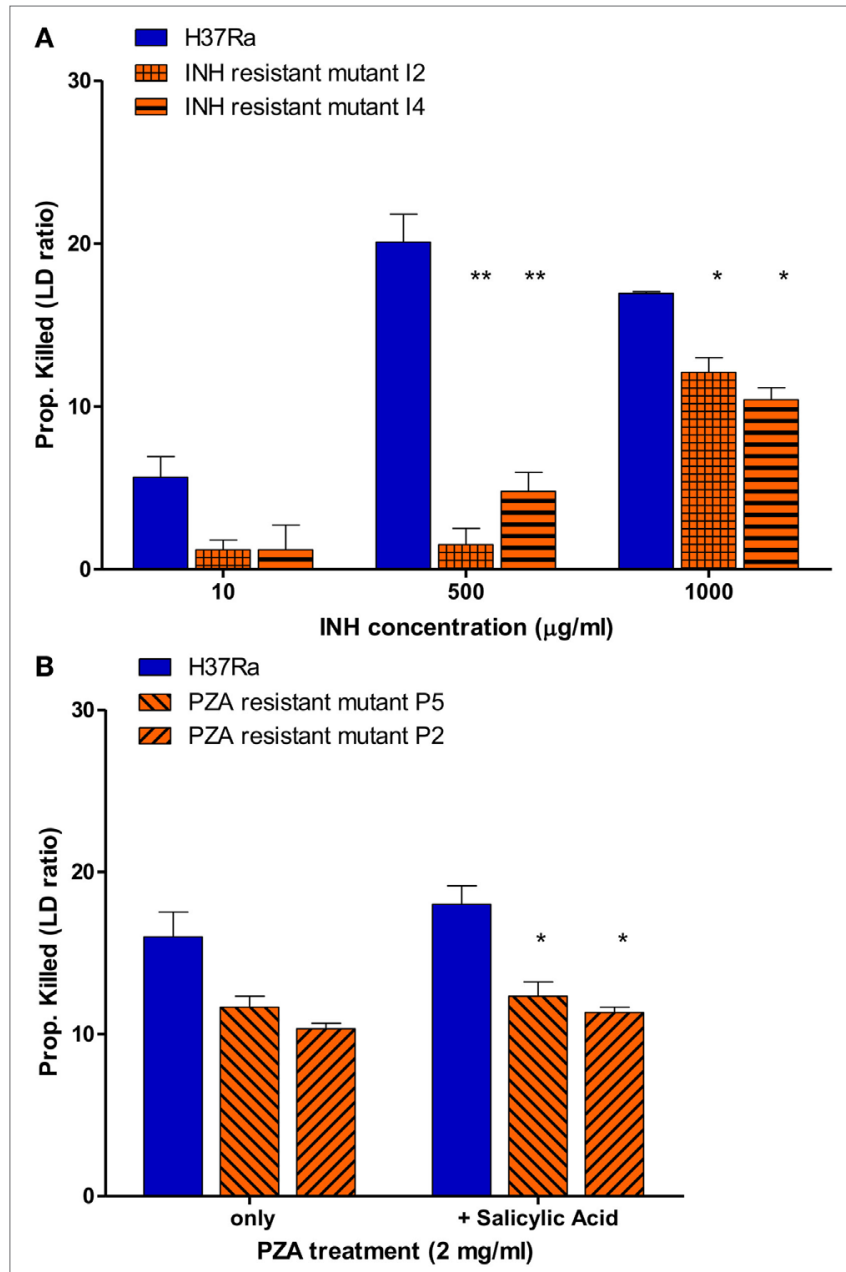

FIGURE 6 | Drug susceptibility testing of Mycobacterium tuberculosis against first-line tuberculosis drugs using the SYBR Green 1/propidium iodide (PI) assay. (A) M. tuberculosis H37Ra and INH-resistant mutants I2, 14 (10-day old) were treated with 10,500, and 1,000 $\mathrm{gg} / \mathrm{ml} \mathrm{INH}$ overnight (16 h). INH-resistant mutants $\mathrm{I} 2$ and 14 were determined to be resistant using 500 and $1,000 \mu \mathrm{g} / \mathrm{ml} \mathrm{INH}$. (B) M. tuberculosis parental strain H37Ra and pyrazinamide (PZA)-resistant mutants (P5 and P2) (20-day old) were treated with PZA (2 mg/ml) overnight (16 h). Treatment with salicylic acid $(40 \mu \mathrm{g} / \mathrm{ml})$ strongly increased the efficacy SYBR Green I/PI assay in detecting PZA resistance in mutants P5 and P2 compared to parental strain H37Ra (Student's $t$-test, ${ }^{\star} p<0.05,{ }^{* *} p<0.005$ ). Data represent the means + SEMs.

PZA susceptible and PZA-resistant strains more clearly than in their absence, allowing for more quantitative measurements of PZA resistance in $M$. tuberculosis.

\section{DISCUSSION}

The current conventional AST relies on the growth of the test organism in the presence of the antibiotic, which can be timeconsuming depending on the growth speed of the organism, ranging from days for fast-growing bacteria to weeks for slow growing bacteria such as $M$. tuberculosis. Here, we developed a rapid and novel antibiotic susceptibility testing methodology using SYBR Green I/PI assay which converts a conventional growth/ culture-dependent AST to a viability-based growth-independent AST. This study is the first to demonstrate the feasibility of the adapted SYBR Green I/PI viability assay to rapidly and reliably detect antibiotic resistance for representative Gram-positive S. aureus, Gram-negative bacteria E. coli, K. pneumoniae in record time of just $30 \mathrm{~min}$, and identify INH and PZA resistance for slow-growing $M$. tuberculosis in just $16 \mathrm{~h}$, which currently takes several weeks. Thus, our newly developed methodology changes the concept of the current antibiotic susceptibility testing and significantly reduces the time needed for the current growthdependent AST.

The automated BACTEC ${ }^{\text {TM }}$ MGITTM 960 (Becton Dickinson, Sparks, MD, USA) is the most commonly used system for $M$. tuberuclosis DST and takes 1-3 weeks, while the Lowenstein-Jensen and 7H10/11 solid media-based DST can take 3-6 weeks, due to the slow growth of the organism. While previous studies have shown that flow cytometry can be used for AST testing for M. tuberculosis in $24 \mathrm{~h}$ (30), the test still relies on growth detection by fluorescence diacetate and has significant drawbacks such as the requirement for expensive instrumentation and lack of high-throughput, and as a result, such method has not been adopted for DST for M. tuberculosis in clinical settings. Despite the high sensitivity achieved by the MBT-ASTRA, as the authors noted, it seems unlikely that MBT-ASTRA will be used in clinical laboratories due to its lack of automated readout (12). Additionally, a luciferase phage system which was developed to detect rifampicin resistance in mycobacteria in 2 days has contamination issues (31). In contrast, the SYBR Green I/PI assay we developed can be used for ultra-rapid DST for not only fast-growing organisms but also for slow-growing organisms like M. tuberculosis in a high-throughput manner such as in 96-well or 384-well plate format, has low detection limits, background noise and more quantitative, and is extremely cost-effective.

It is worth noting that our SYBR Green/PI methodology is different from the current growth-dependent MIC-based AST in that our test used concentrations of antibiotics that are much (10- to 10,000-fold) higher than the MIC values and are chosen based on their ability to distinguish susceptible and resistant strains of the bacterial species tested. We acknowledge that such high concentrations of drugs do not correlate with Cmax values, but such high drug concentrations allow for extremely rapid determination of susceptible versus resistant strains in a record short time. While resistant strains can also be killed in high concentrations of drug, strains KTE181 (E. coli) and NY315 ( $S$. aureus) were characterized as resistant to ampicillin and kanamycin, respectively, by CLSI guidelines showed an average of $0 \%$ of death in our SYBR Green I/PI assay suggesting that higher concentrations of drug used may not always result in killing (Table 1).

The start of an in vitro-cultivation is often associated with the lag phase as adaptation phase of the bacteria to changed growth conditions. This phenomenon could influence the killing of the bacteria by antibiotics and, therefore, the green/red fluorescence ratio. Nevertheless, we were still able to detect a greater proportion 
of dead cells in our susceptible strains for all the organisms tested, presumably due to the high concentrations of antibiotics used. If certain antibiotic does not show good killing on the diluted culture directly, we could potentially enhance the metabolic status of the bacteria by allowing the bacteria to grow for a short time or by adding factors that increase metabolic activity of the bacteria so they respond or become more susceptible to killing by the antibiotic.

It is important to note that our SYBR Green I/PI assay works with several major classes of antibiotics that have different modes of action including inhibitors of cell wall, protein synthesis, DNA gyrase and anti-metabolite agents, and even drugs with unusual mechanisms of action such as PZA. Moreover, our SYBR Green I/PI assay not only works for bactericidal antibiotics (e.g., ampicillin, streptomycin, ciprofloxacin, gentamicin, and kanamycin) but also for bacteriostatic drugs (e.g., trimethoprim, erythromycin, and chloramphenicol) (Figures 2-4). Most importantly for M. tuberculosis, resistance to the critical persister drug PZA, which has been very difficult to determine with current time-consuming growth-based methods, such as MGIT960 (32), can be easily and accurately detected by our SYBR Green/ PI viability assay in just $16 \mathrm{~h}$. Resistance to drugs, such as rifampin, isoniazid, linezolid, and ethambutol, was evaluated in newer MALDI-TOF DST methods but PZA, a first-line drug for treating tuberculosis, has been ignored (12). In fact, the SYBR Green/PI based assay is more appropriate and advantageous for determining the susceptibility of slow growing $M$. tuberculosis to persister drugs like PZA than the conventional MIC-based DST as PZA is mainly active against non-growing persisters with little activity against growing bacilli (27).

There is an increasing antibiotic resistance crisis ongoing and new drugs and antibiotics are urgently needed to combat the life threatening antibiotic-resistant infections. Our newly developed SYBR Green I/PI AST testing methodology, which we developed initially for rapid assessment of viability of B. burgdorferi (21) and subsequently used for high-throughput drug screens against fastidious organism B. burgdorferi persisters (25), can also be adapted for efficient and versatile real-time continuous drug screens to identify new drug candidates targeting both growing and non-growing antibiotic-resistant bacteria, eukaryotic pathogens, and cancer in a high-throughput format.

Despite the potential for the very rapid detection of antibiotic resistance, we are fully aware that future studies to test more clinical strains with different drugs are needed to validate the new SYBR Green I/PI method before implementation in

\section{REFERENCES}

1. Van Rie A, Enarson D. XDR tuberculosis: an indicator of public-health negligence. Lancet (2006) 368(9547):1554-6. doi:10.1016/S0140-6736(06) 69575-5

2. Laxminarayan R, Duse A, Wattal C, Zaidi AK, Wertheim HF, Sumpradit N, et al. Antibiotic resistance-the need for global solutions. Lancet Infect Dis (2013) 13(12):1057-98. doi:10.1016/S1473-3099(13)70318-9

3. Brown ED, Wright GD. Antibacterial drug discovery in the resistance era. Nature (2016) 529(7586):336-43. doi:10.1038/nature17042

4. Zignol M, Dean AS, Falzon D, van Gemert W, Wright A, van Deun A, et al. Twenty years of global surveillance of antituberculosis-drug resistance. N Engl J Med (2016) 375(11):1081-9. doi:10.1056/NEJMsr1512438 the clinic. Future studies include conducting retrospective and prospective studies using a larger collection of strains to validate our breakpoints in determining the different susceptibility categories as characterized in the CLSI guidelines (e.g., resistant, intermediate resistant, and susceptible). Thus, stronger associations between the proportion of dead cells (given by our SYBR Green I/PI assay) and the MIC of a particular drug (from broth dilution methods) can be made so that clinicians can extract more useful clinical information from our assay. However, it is important to note that based on MIC breakpoints from CLSI, S. aureus strains Newman and CA127 are both classified as susceptible to kanamycin despite CA127 having a higher MIC than Newman, 16 and $8 \mu \mathrm{g} / \mathrm{ml}$, respectively. Our SYBR Green I/PI assay was sensitive enough to detect such difference given that the proportion of dead cells in CA127 was lower than Newman, 6.9 and $31.2 \%$, respectively. Additionally, the $95 \%$ confidence interval of resistant and susceptible strains of each particular species do not overlap suggesting that our assay conditions are robust enough to potentially detect differences in MIC.

The goal of our study is to provide a proof of concept of using SYBR Green/PI as a novel and rapid method for detection of antibiotic resistance. In this study, we showed that the SYBR Green/ PI assay can be used for this purpose. Although our studies are on select representative Gram-positive, Gram-negative bacteria, and mycobacteria, this methodology can be adapted to detect resistance in all culturable organisms of prokaryotic and eukaryotic origin for both growing and non-growing cells for both drug susceptibility testing and drug screens in a real-time manner. We anticipate that this new test, subject to further clinical validation, may play important roles in saving lives and improving patient outcomes in a timely manner, as well as identify new drugs for improved treatment of antibiotic-resistant organisms.

\section{AUTHOR CONTRIBUTIONS}

JF and RY contributed equally to this work. YZ, JF, RY, and W-HZ designed the study. JF, RY, SZ, LT, and WS performed the study. JF, RY, W-HZ, and YZ analyzed the data. RY, JF, and YZ wrote the manuscript.

\section{ACKNOWLEDGMENTS}

The authors thank BEI Resources/ATCC for providing strains used in this study.

5. WHO. Global Priority List of Antibiotic-Resistant Bacteria to Guide Research, Discovery, and Development of New Antibiotics. Geneva: World Health Organization (2017).

6. Davies SC, Fowler T, Watson J, Livermore DM, Walker D. Annual Report of the Chief Medical Officer: infection and the rise of antimicrobial resistance. Lancet (2013) 381(9878):1606-9. doi:10.1016/S0140-6736(13)60604-2

7. Goff DA, Kullar R, Goldstein EJC, Gilchrist M, Nathwani D, Cheng AC, et al. A global call from five countries to collaborate in antibiotic stewardship: united we succeed, divided we might fail. Lancet Infect Dis (2017) 17(2): e56-63. doi:10.1016/S1473-3099(16)30386-3

8. Urdea M, Penny LA, Olmsted SS, Giovanni MY, Kaspar P, Shepherd A, et al. Requirements for high impact diagnostics in the developing world. Nature (2006) 444(Suppl 1):73-9. doi:10.1038/nature05448 
9. Kanthor R. Diagnostics: detection drives defence. Nature (2014) 509 (7498):S14-5. doi:10.1038/509S14a

10. CLSI. Performance Standards for Antimicrobial Performance Standards for Antimicrobial Susceptibility Testing-26th Edition: CLSI Supplement M100S. Wayne, PA: NCCLS (2016).

11. CLSI. Susceptibility Testing of Mycobacteria, Nocardiae, and Other Aerobic Actinomycetes-Second Edition M24-A2. Wayne, PA: NCCLS (2011).

12. Ceyssens PJ, Soetaert K, Timke M, Van den Bossche A, Sparbier K, De Cremer K, et al. Matrix-assisted laser desorption ionization-time of flight mass spectrometry for combined species identification and drug sensitivity testing in mycobacteria. J Clin Microbiol (2017) 55(2):624-34. doi:10.1128/ JCM.02089-16

13. Machen A, Drake T, Wang YF. Same day identification and full panel antimicrobial susceptibility testing of bacteria from positive blood culture bottles made possible by a combined lysis-filtration method with MALDITOF VITEK mass spectrometry and the VITEK2 system. PLoS One (2014) 9(2):e87870. doi:10.1371/journal.pone.0087870

14. Idelevich EA, Sparbier K, Kostrzewa M, Becker K. Rapid detection of antibiotic resistance by MALDI-TOF mass spectrometry using a novel directon-target microdroplet growth assay. Clin Microbiol Infect (2017). doi:10.1016/j.cmi.2017.10.016

15. Zignol M, Dean AS, Alikhanova N, Andres S, Cabibbe AM, Cirillo DM, et al. Population-based resistance of Mycobacterium tuberculosis isolates to pyrazinamide and fluoroquinolones: results from a multicountry surveillance project. Lancet Infect Dis (2016) 16(10):1185-92. doi:10.1016/S14733099(16)30190-6

16. Aragon LM, Garrigo M, Moreno C, Espanol M, Coll P. Evaluation of the BacT/ ALERT PZA kit in comparison with the BACTEC 460TB PZA for testing Mycobacterium tuberculosis susceptibility to pyrazinamide. JAntimicrob Chemother (2007) 60(3):655-7. doi:10.1093/jac/dkm252

17. Hewlett D Jr, Horn DL, Alfalla C. Drug-resistant tuberculosis: inconsistent results of pyrazinamide susceptibility testing. JAMA (1995) 273(12):916-7. doi:10.1001/jama.273.12.916

18. Zhang Y, Mitchison D. The curious characteristics of pyrazinamide: a review. Int J Tuberc Lung Dis (2003) 7(1):6-21.

19. Chedore P, Bertucci L, Wolfe J, Sharma M, Jamieson F. Potential for erroneous results indicating resistance when using the Bactec MGIT 960 system for testing susceptibility of Mycobacterium tuberculosis to pyrazinamide. JClin Microbiol (2010) 48(1):300-1. doi:10.1128/JCM.01775-09

20. Simons SO, van Ingen J, van der Laan T, Mulder A, Dekhuijzen PN, Boeree MJ, et al. Validation of pncA gene sequencing in combination with the mycobacterial growth indicator tube method to test susceptibility of Mycobacterium tuberculosis to pyrazinamide. J Clin Microbiol (2012) 50(2):428-34. doi:10.1128/ JCM.05435-11

21. Feng J, Wang T, Zhang S, Shi W, Zhang Y. An optimized SYBR Green I/PI assay for rapid viability assessment and antibiotic susceptibility testing for Borrelia burgdorferi. PLoS One (2014) 9(11):e111809. doi:10.1371/journal. pone.0111809
22. Barbesti S, Citterio S, Labra M, Baroni MD, Neri MG, Sgorbati S. Two and three-color fluorescence flow cytometric analysis of immunoidentified viable bacteria. Cytometry (2000) 40(3):214-8. doi:10.1002/1097-0320(20000701) 40:3<214::AID-CYTO6>3.0.CO;2-M

23. Zhang Y, Yew WW. Mechanisms of drug resistance in Mycobacterium tuberculosis. Int J Tuberc Lung Dis (2009) 13(11):1320-30.

24. Zhang S, Chen J, Shi W, Liu W, Zhang W, Zhang Y. Mutations in panD encoding aspartate decarboxylase are associated with pyrazinamide resistance in Mycobacterium tuberculosis. Emerg Microbes Infect (2013) 2(6):e34. doi:10.1038/emi.2013.38

25. Feng J, Wang T, Shi W, Zhang S, Sullivan D, Auwaerter PG, et al. Identification of novel activity against Borrelia burgdorferi persisters using an FDA approved drug library. Emerg Microbes Infect (2014) 3(7):e49. doi:10.1038/ emi.2014.53

26. Zhang Y, Chiu Chang K, Leung CC, Wai Yew W, Gicquel B, Fallows D, et al. 'Z(S)-MDR-TB' versus ' $\mathrm{Z}(\mathrm{R})-\mathrm{MDR}-\mathrm{TB}$ ': improving treatment of MDR-TB by identifying pyrazinamide susceptibility. Emerg Microbes Infect (2012) 1(7):e5. doi:10.1038/emi.2012.18

27. Zhang Y, Shi W, Zhang W, Mitchison D. Mechanisms of pyrazinamide action and resistance. Microbiol Spectr (2014) 2(4). doi:10.1128/microbiolspec. MGM2-0023-2013

28. Zhang Y, Scorpio A, Nikaido H, Sun Z. Role of acid $\mathrm{pH}$ and deficient efflux of pyrazinoic acid in unique susceptibility of Mycobacterium tuberculosis to pyrazinamide. J Bacteriol (1999) 181(7):2044-9.

29. Wade MM, Zhang Y. Effects of weak acids, UV and proton motive force inhibitors on pyrazinamide activity against Mycobacterium tuberculosis in vitro. J Antimicrob Chemother (2006) 58(5):936-41. doi:10.1093/jac/dkl358

30. Fredricks BA, DeCoster DJ, Kim Y, Sparks N, Callister SM, Schell RF. Rapid pyrazinamide susceptibility testing of Mycobacterium tuberculosis by flow cytometry. J Microbiol Methods (2006) 67(2):266-72. doi:10.1016/j. mimet.2006.03.020

31. Mole R, Trollip A, Abrahams C, Bosman M, Albert H. Improved contamination control for a rapid phage-based rifampicin resistance test for Mycobacterium tuberculosis. J Med Microbiol (2007) 56(Pt 10):1334-9. doi:10.1099/jmm.0.46936-0

32. Mitchison DA. The action of antituberculosis drugs in short-course chemotherapy. Tubercle (1985) 66(3):219-25. doi:10.1016/0041-3879(85)90040-6

Conflict of Interest Statement: A patent application (\# US 15/347,285, EP17159022.7-1405, CN201580037580.4) pertaining to the results presented in the paper was filed.

Copyright $\odot 2018$ Feng, Yee, Zhang, Tian, Shi, Zhang and Zhang. This is an openaccess article distributed under the terms of the Creative Commons Attribution License (CC BY). The use, distribution or reproduction in other forums is permitted, provided the original author(s) and the copyright owner are credited and that the original publication in this journal is cited, in accordance with accepted academic practice. No use, distribution or reproduction is permitted which does not comply with these terms. 